\title{
Soroprevalência de anticorpos para toxoplasmose, rubéola, citomegalovírus, sífilis e HIV em gestantes sergipanas
}

\author{
Seroprevalence of antibodies for toxoplasmosis, rubella, cytomegalovirus, \\ syphilis and HIV among pregnant women in Sergipe
}

\author{
Ana Dorcas de Melo Inagaki ${ }^{1,2}$, Lívia Albuquerque Resende de Oliveira ${ }^{3}$, \\ Maria Fabiana Batista de Oliveira ${ }^{3}$, Ricardo Cley Silvestre Santos ${ }^{3}$, Raquel Melo Araújo ${ }^{3}$, \\ José Antonio Barreto Alves ${ }^{4}$, Kariny Souza Pinheiro ${ }^{5}$, Ricardo Queiroz Gurgel ${ }^{2}$ \\ e Marisa Márcia Mussi-Pinhata ${ }^{6}$
}

\begin{abstract}
RESUMO
Averiguou-se a soroprevalência para HIV, sífilis, toxoplasmose, citomegalovirose e rubéola em gestantes de Sergipe, nordeste do Brasil, verificando-se a associação com idade e procedência. Selecionaram-se 9.550 gestantes ( 2.112 da capital e 7.438 do interior) testadas consecutivamente durante o primeiro atendimento pré-natal em 2007. Foram encontradas as seguintes frequências de soropositividade: sífilis (0,9\%; IC95\% 0,7\%-1,6\%), HIV (0,14\%; IC95\% 0,08\%-0,2\%), toxoplasmose (IgG 69,3\%; IC95\% 68,3\%-70,2\%; IgM 0,4\%, IC95\% 0,3\%-0,6\%), citomegalovirose (IgG 76,6\%, IC95\% 75,7\%-77,5\%; IgM 0,2\%, IC95\% 0,09\%-0,3\%) e rubéola (IgG 71,6\%, IC95\% 70,7\%-72,6\%; IgM 0,1\%, IC95\% 0,04\%-0,2\%). A soropositividade para toxoplasmose incrementou com a idade. A prevalência de anticorpos IgG para toxoplasmose, CMV e rubéola foi maior na capital, Aracaju, que nos demais municípios do Estado de Sergipe. Foi encontrada associação entre a idade e soropositividade para a toxoplasmose. Os achados revelam grande proporção de gestantes susceptíveis a toxoplasmose, rubéola e citomegalovirose, principalmente no interior do estado, com risco para seus filhos.
\end{abstract}

Palavras-chaves: Soroprevalência. Infecção congênita. Gestante. Pré-natal. Sergipe.

\begin{abstract}
The seroprevalence of antibodies for HIV, syphilis, toxoplasmosis, cytomegalovirosis and rubella and its association with age and origin was investigated among pregnant women in Sergipe, northeastern Brazil. A total of 9,550 pregnant women (2,112 from the state capital and 7,438 from other municipalities) were enrolled in the study and consecutively tested during their first antenatal care visit in 2007. The following serum prevalences were found: syphilis (0.9\%; 95\% CI 0.7\%-1.6\%), HIV (0.14\%; 95\% CI 0.08\%-0.2\%), toxoplasmosis (IgG 69.3\%; 95\% CI 68.3\%-70.2\%; IgM 0.4\%, 95\% CI 0.3\%-0.6\%), cytomegalovirosis (IgG 76.6\%, 95\% CI 75.7\%-77.5\%; IgM 0.2\%, 95\% CI 0.09\%-0.3\%) and rubella (IgG 71.6\%, 95\% CI 70.7\%-72.6\%; IgM 0.1\%, $95 \%$ CI $0.04 \%-0.2 \%$ ). Toxoplasmosis seropositivity increased with age. The prevalences of $\operatorname{IgG}$ antibodies for toxoplasmosis, cytomegalovirus and rubella were higher in Aracaju (state capital) than in other municipalities in the State of Sergipe. The results showed that a large proportion of the pregnant women, particularly in municipalities other than the state capital, were susceptible to toxoplasmosis, rubella and cytomegalovirus, with a risk for their infants.
\end{abstract}

Key-words: Seroprevalence. Congenital infection. Pregnant women. Prenatal. Sergipe.

Conhecer a soroprevalência gestacional de agentes que possam ser transmitidos da mãe para o feto e causar doença é uma ferramenta essencial para formulação de políticas públicas de saúde, favorecendo o planejamento de ações programáticas de prevenção e assistência.

\footnotetext{
1. Curso de Pós-Graduação em Saúde da Criança e do Adolescente, Faculdade de Medicina de Ribeirão Preto, Universidade de São Paulo, Ribeirão Preto, SP. 2. Universidade Federal de Sergipe, Aracaju, SE. 3. Acadêmico do Curso de Enfermagem, Universidade Federal de Sergipe, Aracaju, SE 4. Curso de Pós-Graduação em Ciências da Saúde, Universidade Federal de Sergipe, Aracaju, SE. 5. Programa de Proteção à Gestante, Laboratório Central de Sergipe (LACEN), Aracaju, SE. 6. Faculdade de Medicina de Ribeirão Preto, Universidade de São Paulo, Ribeirão Preto, SP. Endereço para correspondência: Dra. Ana Dorcas de Melo Inagaki. Rua Duque de Caxias 167/1202, Bairro São José, 49015-320 Aracaju, SE.

Tel: 5579 9999-3769; 55 79 3221-1139

e-mail: anadorcas@usp.br; laurodorcas@oi.com.br

Recebido para publicação em 30/03/2009

Aceito em 29/09/2009
}

Em Sergipe, o serviço de assistência pré-natal realiza, rotineiramente, testes sorológicos de triagem para toxoplasmose, rubéola, sífilis, citomegalovírus (CMV) e vírus da imunodeficiência humana (HIV) em todas as gestantes atendidas pelo Sistema Único de Saúde.

Este estudo visou conhecer a soroprevalência para toxoplasmose, rubéola, CMV, sífilis e HIV no estado de Sergipe e verificar se havia associação entre a idade e a procedência com a sopositividade para esses agentes, uma vez que não havia informações disponíveis sobre a ocorrência dessas infecções em gestantes desse estado.

\section{MATERIAL E MÉTODOS}

Estudo populacional, epidemiológico, de corte transversal, realizado por meio da análise de dados de forma retrospectiva. 
O projeto foi aprovado pelo Comitê de Ética em Pesquisa com Seres Humanos da Universidade Federal de Sergipe (processo CAAE- 4192.0.000.107-07).

Foram avaliados os resultados de testes sorológicos de triagem para detecção de anticorpos da classe IgG e IgM para toxoplasmose, rubéola, CMV, assim como detecção de anticorpos totais para sífilis e HIV de todas as gestantes atendidas pelo Sistema Único de Saúde (SUS), durante o quadrimestre de julho a outubro de 2007, sendo 2.112 gestantes residentes na capital de Sergipe, Aracaju, e 7.438 gestantes provenientes dos demais 74 municípios sergipanos, totalizando 9.550 mulheres. Foram avaliados os 9.550 registros médicos que continham os resultados dos exames realizados após primeiro atendimento pré-natal na unidade básica de saúde.

Para análise dos dados, foram considerados somente os resultados que puderam ser definidos por meio do teste de triagem, excluindo-se aqueles com resultados indeterminados, sendo 334 IgG e 77 IgM para toxoplasmose; 349 IgG e159 IgM para CMV, 611 IgG e 47 IgM para rubéola além de 47 para VDRL). Dos exames de HIV, nenhum se revelou inconclusivo.

Para avaliação da associação entre a soroprevalência e a idade, foram excluídas 499 (5,2\%) mulheres, pois a informação sobre a idade das mesmas não estava disponível nos registros.

Os testes sorológicos das gestantes provenientes de Aracaju foram realizados em amostra de sangue periférico. Para esses ensaios sorológicos, realizados no Centro de Especialidades Médicas de Aracaju (CEMAR), foram utilizados os seguintes testes, obedecendo-se às instruções dos fabricantes: bioelisa Toxo IgG e IgM, bioelisa Rubella IgG e IgM, bioelisa CMV IgG e IgM, BIOKIT, S.A.Lliçà d'Amunt, Barcelona, SPAIN ${ }^{\circledR}$. Para sífilis, foi utilizado o VDRL (Wiener laboratórios S.A.I.C. Rosario-Argentina) e para HIV o teste Ultra HIV Ag-Ab (GENSCREEN ${ }^{\circledR}$, Marnes-laCoquette - France).

Das gestantes provenientes dos demais 74 municípios do Estado de Sergipe, foi obtida amostra de sangue absorvido em papel filtro (S\&S 903 Symbiosis Diagnóstica Ltda, São Paulo, Brasil). Os testes sorológicos foram realizados no Instituto Parreira Horta (HEMOLACEN-SE), utilizando-se os seguintes ensaios, segundo as recomendações do fabricante: Q-Preven Toxo IgG e IgM - DBS, Q-Preven CMV IgG e IgM - DBS, rubéola IgG e IgM - DBS, Q-Preven Sífilis Total - DBS e Q-Preven HIV 1+2-DBS, fabricados por Symbiosis Diagnóstica Ltda, São Paulo, Brasil.

Os dados obtidos foram processados no pacote estatístico Epi info (Epi 3.4 e Epi6 - Centers for Disease Control and Prevention - Atlanta, EUA). Para avaliar a associação entre prevalência de anticorpos para essas infecções e a faixa etária e/ou procedência, foi aplicado o teste do qui-quadrado e teste exato de Fisher. Para medir a força da associação entre essas variáveis foi realizada a regressão logística, utilizando-se o PROC logistic, do software SAS 9.1 (SAS Institute Inc, Cary, NC, USA). Utilizando-se o teste de $t$ de Student bicaudal para duas amostras independentes, comparou-se a idade das gestantes segundo a procedência.

\section{RESULTADOS}

Na ocasião da coleta de sangue para realização da sorologia a idade foi informada por 9.051 gestantes. A média de idade das 9.051 gestantes estudadas foi de 24,5 anos (10 a 53 anos), sendo a proporção de adolescentes (10 a 19 anos) de 24,9\%. As gestantes assistidas no interior do estado eram mais jovens (24,4 anos) do que aquelas assistidas na capital (25,2 anos) $(\mathrm{p}<0,01)$.

A soroprevalência para cada um dos agentes investigados segundo a categoria de idade pode ser observada na Tabela 1. Foi detectada associação entre a idade e soroprevalência para testes de VDRL, observando-se maior positividade entre as gestantes com idade entre 20 a 34 anos. Quanto à soropositividade para anticorpos IgG antitoxoplasma, foi observado incremento na soroprevalência com o aumento da idade. Não foi encontrada associação entre a idade e a soropositividade para demais anticorpos pesquisados.

Quanto à procedência, foram encontradas significativas taxas de prevalência para anticorpos IgG contra o Toxoplasma gondii, o vírus da rubéola e o citomegalovírus, maior na capital, Aracaju, mesmo após o ajuste realizado por meio da regressão logística para a variável idade materna, como mostra a Tabela 2.

TABELA 1

Taxa de soroprevalência em percentagem para sífilis, HIV, toxoplasmose, CMV e rubéola entre gestantes sergipanas de acordo com a faixa etária, no período de julho a outubro de 2007 .

\begin{tabular}{|c|c|c|c|c|}
\hline & \multicolumn{3}{|c|}{ Faixa etária (anos) } & \multirow[b]{2}{*}{ Total } \\
\hline & 10 a 19 & 20 a 34 & $\geq 35$ & \\
\hline \multirow[t]{2}{*}{ Sífilis $\epsilon^{€}$} & 0,4 & 1,2 & 0,9 & 0,9 \\
\hline & {$[0,2-0,7]$} & {$[0,9-1,5]$} & {$[0,4-1,8]$} & {$[0,7-1,6]$} \\
\hline \multirow[t]{2}{*}{ HIV } & 0,09 & 0,18 & - & 0,14 \\
\hline & {$[0,01-0,3]$} & {$[0,09-0,3]$} & - & {$[0,08-0,2]$} \\
\hline \multirow[t]{2}{*}{ Toxoplasma gondii (IgM) } & 0,6 & 0,4 & 0,3 & 0,4 \\
\hline & {$[0,3-1,0]$} & {$[0,3-0,6]$} & {$[0,04-1,0]$} & {$[0,3-0,6]$} \\
\hline \multirow{2}{*}{ Toxoplasma gondii $(\mathrm{IgG})$} & )$^{€} 67,2$ & 69,6 & 73,1 & 69,3 \\
\hline & {$[65,1-69,1]$} & {$[68,4-70,7]$} & {$[69,7-76,2]$} & {$[68,3-70,2]$} \\
\hline \multirow[t]{2}{*}{ CMV (IgM) } & 0,3 & 0,1 & - & 0,2 \\
\hline & {$[0,1-0,7]$} & {$[0,05-0,2]$} & - & {$[0,09-0,3]$} \\
\hline \multirow[t]{2}{*}{ CMV (IgG) } & 76,0 & 76,8 & 77,1 & 76,6 \\
\hline & {$[74,1-77,8]$} & {$[75,7-77,8]$} & {$[73,9-80,0]$} & {$[75,7-77,5]$} \\
\hline \multirow[t]{2}{*}{ Rubéola (IgM) } & 0,04 & 0,1 & 0,3 & 0,1 \\
\hline & {$[0,002-0,3]$} & {$[0,03-0,2]$} & {$[0,04-1,0]$} & {$[0,04-0,2]$} \\
\hline \multirow[t]{2}{*}{ Rubéola (IgG) } & 71,2 & 71,8 & 71,5 & 71,6 \\
\hline & {$[69,2-73,2]$} & {$[70,6-72,9]$} & {$[68,1-74,7]$} & {$[70,7-72,6]$} \\
\hline
\end{tabular}

HIV: vírus da imunodeficiência humana, CMV: citomegalovírus. IC95\%] ${ }^{€} \mathrm{p}=0,007$. IC95\%: intervalo de confiança de $95 \%$ 


\section{TABELA 2}

Taxa de soroprevalência em percentagem de testes para sífilis, HIV, toxoplasmose, CMV e rubéola entre gestantes sergipanas, no período de julho a outubro de 2007 , segundo a procedência.

\begin{tabular}{|c|c|c|c|}
\hline & $\begin{array}{c}\text { Soroprevalência (\%) } \\
\text { [IC95\%] }\end{array}$ & $\begin{array}{c}\text { Odds ratio } \\
\text { ajustado para a } \\
\text { idade }\end{array}$ & IC95\% \\
\hline \multicolumn{4}{|l|}{ Sífilis } \\
\hline demais municípios & $0,80[0,7-1,1]$ & 1,0 & \\
\hline Aracaju & $1,09[0,7-1,6]$ & 1,16 & $0,72-1,88$ \\
\hline \multicolumn{4}{|l|}{ HIV } \\
\hline demais municípios & $0,17[0,1-0,3]$ & 1,0 & \\
\hline Aracaju & $0,14[0,1-0,3]$ & 0,81 & $0,18-3,04$ \\
\hline \multicolumn{4}{|c|}{ Toxoplasmosi gondii (IgM) } \\
\hline demais municípios & $0,52[0,4-0,7]$ & 2,04 & $0,80-5,24$ \\
\hline Aracaju & $0,23[0,1-0,6]$ & 1,0 & \\
\hline \multicolumn{4}{|c|}{ Toxoplasmosi gondii (IgG) } \\
\hline demais municípios & $66,3[65,1-67,4]$ & 1,0 & \\
\hline Aracaju & $77,8[75,9-79,5]$ & 1,75 & $1,56-1,96$ \\
\hline \multicolumn{4}{|l|}{ CMV (IgM) } \\
\hline demais municípios & $0,13[0,1-0,3]$ & 1,0 & \\
\hline Aracaju & $0,19[0,1-0,5]$ & 1,40 & $0,43-4,58$ \\
\hline \multicolumn{4}{|l|}{ CMV (IgG) } \\
\hline demais municípios & $74,0[72,9-75,0]$ & 1,0 & \\
\hline Aracaju & $84,3[82,6-85,8]$ & 1,86 & $1,62-2,12$ \\
\hline \multicolumn{4}{|l|}{ Rubéola (IgM) } \\
\hline demais municípios & $0,08[0,03-0,18]$ & 1,0 & \\
\hline Aracaju & $0,09[0,01-0,31]$ & 1,04 & $0,21-5,18$ \\
\hline \multicolumn{4}{|l|}{ Rubéola (IgG) } \\
\hline demais municípios & $64,3[63,2-65,5]$ & 1,0 & \\
\hline Aracaju & $92,8[91,6-93,8]$ & 7,08 & $5,96-8,42$ \\
\hline
\end{tabular}

HIV: vírus da imunodeficiência humana, CMV: citomegalovírus, IC95\%: intervalo de confiança de $95 \%$

\section{DISCUSSÃo}

Este estudo veio suprir uma lacuna do conhecimento de dados relevantes para o planejamento e promoção de saúde perinatal no Estado de Sergipe, ao verificar a soroprevalência para diferentes agentes infecciosos em amostra representativa de gestantes provenientes de todas as regiões do estado.

Apesar deste estudo apresentar limitações inerentes a um estudo de prevalência realizado de forma retrospectiva, que empregou diferentes metodologias laboratoriais de triagem e não terem sido feitos testes confirmatórios, o grande tamanho amostral e sua representatividade com relação à população de gestantes sergipanas, o tornam adequado para a estimativa que se pretendeu. Além de ter demonstrado que a soroprevalência em gestantes assistidas no Sistema Único de Saúde é semelhante à maioria dos dados brasileiros disponíveis, identificamos maior soroprevalência para toxoplasmose, citomegalovirose e rubéola entre as gestantes residentes na capital do que no interior do estado.

A menor prevalência de anticorpos antitoxoplasma, encontrada em estudos epidemiológicos brasileiros, foi de
41,9\%, em Florianópolis, $\mathrm{SC}^{1}$, enquanto a maior prevalência foi de 91,6\%, encontrada no Mato Grosso do Sul ${ }^{45}$. A prevalência de anticorpos da classe IgG antitoxoplasma em Sergipe $(69,3 \%)$, está de acordo com a maioria dos estudos brasileiros ${ }^{139192122232526}$, revelando ser freqüente a exposição ao protozoário nessa população. Todavia ainda há grande proporção $(30,7 \%)$ de gestantes susceptíveis, o que revela a necessidade de instituição de medidas que visem à divulgação universal de informações sobre prevenção da toxoplasmose durante a gestação, para que se evite a transmissão vertical do protozoário. Quanto à detecção de anticorpos da classe IgM, que poderiam estimar, levando em consideração as limitações já reconhecidas ${ }^{812}$, a ocorrência de infecção recente pelo toxoplasma, nossos achados são também semelhantes a outros estudos brasileiros ${ }^{5024}$. Porém, de menor frequência que as encontradas na década de 1990, por Olbrich Neto e Meira $(2,1 \%)^{16}$ e, no início dos anos 2000 , por Reis e cols ${ }^{21}(2,7 \%)$.

Corroborando o conhecimento já existente de que há incremento da prevalência de anticorpos contra o toxoplasma à medida que aumenta a idade, observamos maior prevalência de anticorpos antitoxoplasma da classe IgG entre as gestantes com 35 anos ou mais do que entre aquelas das menores faixas etárias. Merece ainda atenção 0 achado da maior chance de soropositividade para toxoplasmose, independentemente da idade, nas gestantes residentes na cidade de Aracaju, do que em outras cidades do estado. Outros estudos devem ser realizados para identificação das fontes de infecção, se relacionadas a hábitos alimentares, condições sanitárias e/ou higiênicas nas diferentes populações.

Estudos sobre soroprevalência para CMV em gestantes brasileiras relatam proporções de $66,5 \%$ em populações de elevado nível socioeconômico e 84,4 a 95\% em populações de baixo nível socioeconômico ${ }^{711}{ }^{18}$. De maneira geral, nossos achados (76,6\%) se aproximam daqueles de populações de menor nível socioeconômico. Curiosamente, maior freqüiência de detecção de anticorpos IgG, independentemente da idade, foi observada na capital $(84,3 \%)$, do que no interior do estado (74\%), sugerindo haver diferenças populacionais quanto aos fatores facilitadores da infecção por esse vírus ${ }^{6}$.

Considerando-se que a infecção congênita pelo CMV possa ser consequiência tanto da infecção primária quanto da infecção secundária materna durante a gestação e, que maior é o risco de infecção congênita quanto maior a soropositividade materna ${ }^{7}, 0$ conhecimento da elevada soropositividade, associada a grande proporção de mulheres susceptíveis, permite estimar que a ocorrência de infecção congênita por esse vírus nessa população seja frequente. Provavelmente, esta se assemelhe àquela encontrada em estudo que realizou triagem neonatal em grande número de crianças na região sudeste do Estado de São Paulo, o qual identificou $1 \%$ de recém-nascidos infectados, independentemente da presença de manifestações de infecção congênita ${ }^{15}$.

Surpreendentemente, apesar da soropositividade para rubéola não ter variado com a idade, a chance que gestantes residentes na capital fossem soropositivas para anticorpos da classe IgG foi de 7 vezes aquela encontrada para gestantes dos demais municípios, indicando que o estado de imunização natural e/ 
ou ativa das mulheres em idade fértil difere muito entre as populações. Enquanto 36\% das gestantes do interior do estado eram suscetíveis ao vírus da rubéola, somente $7 \%$ daquelas da capital o eram. Dados relativos à vacinação contra rubéola dessas regiões que indicam menor cobertura vacinal nos municípios do interior do que na capital ${ }^{14}$, podem explicar nossos achados.

Embora a soropositividade para sífilis por nós detectada tenha sido menor que a média nacional $(1,6 \%)^{17}$ ela foi semelhante à encontrada no Mato Grosso do $\operatorname{Sul}^{5}(0,92 \%)$. No entanto, a prevalência entre as gestantes com idade entre 20 a 34 anos, por nós avaliadas, foi próxima à média nacional, possivelmente revelando a maior exposição e risco de aquisição da infecção nessa faixa etária, na qual ocorre a maioria das gestações.

A prevalência de soropositividade para HIV encontrada em nosso estudo está de acordo com dados nacionais observados no estudo sentinela ${ }^{13}$ que estima ser de $0,41 \%$, com menores frequiências, no norte e nordeste, do que nas demais regiões brasileiras. No entanto, deve-se considerar que devido ao fato de gestantes sergipanas sabidamente portadoras do HIV realizarem pré-natal em centro de referência e não serem incluídas nos programas de triagem pré-natal rotineiros é provável que, a prevalência por nós detectada esteja subestimada com relação à verdadeira prevalência populacional. Dados obtidos por estudo realizado em maternidades sergipanas em $2004^{10}$, o qual identificou prevalência de $0,41 \%$, corroboram essa possibilidade, pois no citado estudo, $41 \%$ das gestantes infectadas pelo HIV já conheciam o seu estado de infecção.

Em conclusão, devido à possibilidade de profilaxia e tratamento gestacional, com indiscutível benefício para mãe, feto e recém-nascido, sob risco de aquisição de infecção causada pelo HIV e Treponema pallidun e à chance de prevenção de infecção gestacional a partir do conhecimento da soronegatividade para toxoplasmose, as prevalências por nós encontrada em gestantes do Estado de Sergipe, justificam plenamente a manutenção da testagem sorológica para esses agentes. No entanto, tendo em vista que não há, até o momento, nenhuma modalidade de tratamento materno que previna ou reduza a chance de ocorrência de infecção ou doença fetal que seja dependente do conhecimento do estado sorológico pré-natal, acreditamos que os recursos utilizados para a realização dos testes para CMV pudessem ser mais bem aplicados em campanhas de divulgação das medidas de prevenção da aquisição da infecção gestacional por meio de orientações a todas as gestantes, quanto aos hábitos higiênicos no contato com outros adultos ou crianças. Também entendemos que os recursos utilizados na testagem para rubéola devessem ser alocados para vacinação de mulheres susceptíveis.

\section{REFERÊNCIAS}

1. Cantos GA, Prando MD, Siqueira MV, Teixeira RM. Toxoplasmose: ocorrência de anticorpos antiToxoplasma gondii e diagnóstico. Revista da Associação Médica Brasileira 46: 335-341 2000 .

2. Duarte G, Kawasaki E, Figueiredo LTM, Yamamoto AY, Carlucci RM. Estudo soroepidemiológico sobre citomegalovírus em parturientes e recém-nascidos. Revista Brasileira de Ginecologia e Obstetrícia 16: 153-158, 1994.
3. Duarte G, Paschoini MC, Martinez R, Ramos DM, Turco F. Avaliação soroepidemiológica de toxoplasmose em puérperas. Revista Brasileira de Ginecologia e Obstetrícia. In: $48^{\circ}$ Congresso Brasileiro de Ginecologia e Obstetrícia. Goiânia, p 175, 1999.

4. Figueiró-Filho EA, Lopes AHA, Senefonte FRA, Souza Júnior VG, Botelho CA, Figueiredo MS, Duarte G. Toxoplasmose aguda: estudo da frequiência, taxa de transmissão vertical e relação entre os testes diagnósticos materno-fetais em gestantes em estado da Região Centro-Oeste do Brasil. Revista Brasileira de Ginecologia e Obstetrícia. 8: 442-449, 2005.

5. Figueiró-Filho EA, Senefonte FRA, Lopes AHA, Morais 00, Souza Júnior VG, Maia TL, Duarte G. Frequiência das infecções pelo HIV-1, rubéola, sífilis, toxoplasmose, citomegalovírus, herpes simples, hepatite $\mathrm{B}$, hepatite $\mathrm{C}$, doença de Chagas e HTLV I/II em gestantes, do estado do Mato Grosso do Sul. Revista da Sociedade Brasileira de Medicina Tropical 40: 181-187 2007.

6. Fowler KB, Pass RF. Risk factores for congenital cytomegalovirus infection in the offspring of Young women: exposure to Young children and recent onset of sexual activity. Pediatrics 118: 286-292, 2006.

7. Gaytant MA, Steegers EA, Semmekront BA, Merkus HM, Galama JM. Congenital cytomegalovirus infection: review of the epidemiology and outocome. Obstetrical \& Gynecological Survey 57: 245-256, 2002.

8. Gras L, Gilbert RE, Wallon M, Peyron F, Cortina-BorjaM. Duration of the IgM response in women acquiring Toxoplasma-gonddi during pregnancy: implications for clinical and cross-sectional incidence studies. Epidemiology and Infection 132: 541-548, 2004.

9. Inagaki ADM. Toxoplasmose e Gravidez. Dissertação de Mestrado: Universidade Federal de São Paulo - UNIFESP, São Paulo, 1998.

10. Lemos LMD, Gurgel RQ, Fabbro ALD. Prevalência da infecção por HIV em parturientes de maternidades vinculadas ao SUS. Revista Brasileira de Ginecologia e Obstetrícia 27: 32-36 2005.

11. Machado CM, Fink MC, Vilas-Boas LS, Sumita LM, Weinberg A, Shiguematsu K, Souza IC, Casanova LD, Pannuti CS. Perinatal infection due to cytomegalovirus in a public hospital of the municipality of São Paulo: a pospective study. Revista do Instituto de Medicina Tropical. São Paulo. 33: 159-166, 1991.

12. Meek B, GoolRE, Gilis H, PeekR. Dissecting The IgM antibody response during the acute and latent phase of toxoplasmosis. Diagnostic Microbiology \& Infectious Disease 41: 131-137 2001.

13. Ministério da Saúde do Brasil. Coordenação Nacional de DST/AIDS. Disponível em HTTP//www.aids.gov.br/services/DocumentManagement/FileDownload.EZTSvc. asp? Acessado em 09 de fevereiro de 2009.

14. Ministério da Saúde do Brasil. Programa nacional de Imunização. Disponível em HTTP//www pni.datasus.gov.br/consulta_rubeola_mun_br_08.asp? Acessado em 09 de fevereiro de 2009.

15. Mussi-Pinhata MM, Yamamoto AY, Brito RMM, Isaac ML, Boppana S, Britt WJ. Birth Prevalence and Natural History of Congenital Cytomegalovirus (CMV) Infection in a Highly Seroimmune Population. Clinical infectious diseases 49: 522-528 2009 .

16. Olbrich NJ, Meira DA. Soroprevalência de vírus linfotrópico de células T humanas, vírus da imunodeficiência humana, sífilis e toxoplasmose em gestantes de Botucatu (SP) - Brasil. Fatores de risco para vírus linfotrópico de células T humana. Revista da Sociedade Brasileira de Medicina Tropical 37: 28-29 2004.

17. Organização Não Governamental ELIMINASIFILIS 2007- SETOR DST/UFF. Disponível em: <http://www.eliminasifilis.org/sc_brasil.php>. Acesso em: 11 de julho de 2008.

18. Pannuti CS, Vilas-Boas LS, Angelo MJ, Carvalho RP, Segre CM. Congenital cytomegalovirus infection. Ocorrence in two socioeconomically distinct population of a developing country. Revista do Instituto de Medicina Tropical São Paulo 27: 105-107, 1985.

19. Porto AMF, Amorim MMR, Coelho ICN, Santos LC. Perfil sorológico para toxoplasmose em gestantes atendidas em maternidade. Revista da Associação Médica Brasileira 54: 242-248 2008.

20. Reiche EMV, Morimoto HK, Farias GN, Hisatsugu KR, Geller L, Gomes ACLF, Inoue HY, Rodrigues G, Matsuo T. Prevalência de tripanossomíase americana, sífilis, toxoplasmose, rubéola, hepatite $\mathrm{B}$, hepatite $\mathrm{C}$ e da infecção pelo vírus da imunodeficiência humana avaliada através de testes sorológicos, em gestantes 
atendidas no período de 1996 a 1998 no Hospital Universitário Regional Norte do Paraná (Universidade Estadual de Londrina, Paraná, Brasil). Revista da Sociedade Brasileira de Medicina Tropical 33: 519-527, 2000

21. Reis MM, Tessaro MM, d'Azevedo PA. Perfil sorológico para toxoplasmose em gestantes de um hospital público de Porto Alegre. Revista Brasileira de Ginecologia e Obstetrícia 3: 158-164 2006.

22. Rey LC, Ramalho ILC. Seroprevalence of toxoplasmosis in Fortaleza, Ceará, Brazil. Revista do Instituto de Medicina Tropical São Paulo 41: 171-174 1999.

23. Santos JL, Lopes MAA, Deliege-Vasconcelos E, Couto-Fernandez JC, Patel BN, Barreto ML, Ferreira Jr OC, Galvão-Castro B. Seroprevalence of HIV, HTLV-IVI and other perinatally-transmitted pathogens in Salvador, Bahia. Revista do Instituto de Medicina Tropical São Paulo 37: 343-348 1995.

24. Segundo GRS, Silva DAO, Mineo JR, Ferreira MS. A comparative study of congenital toxoplasmosis between public and private hospitals from Uberlândia, MG, Brazil. Memórias do Instituto Oswaldo Cruz 99: 13-17, 2004.

25. Spalding SM, Amendoeira MRR, Ribeiro LC, Silveira C, Garcia AP, Camilo-Coura L. Estudo prospectivo de gestantes e seus bebês com risco de transmissão de toxoplasmose congênita em município do Rio Grande do Sul. Revista da Sociedade Brasileira de Medicina Tropical 36: 483-491 2003.

26. Varella IS, Wagner MB, Darela AC, Nunes LM, Müller RW. Prevalência de soropositividade para toxoplasmose em gestantes. Journal Pedriatric 79: 69-74 2003. 